\title{
On the ionospheric perturbation associated with the 2007 Niigata Chuetsu-oki earthquake, as seen from subionospheric VLF/LF network observations
}

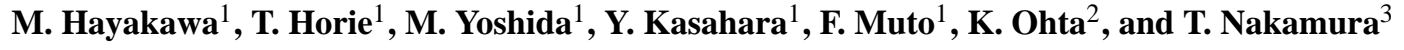 \\ ${ }^{1}$ Department of Electronic Engineering and Research Station on Seismo Electromagnetics, The University of \\ Electro-Communications, 1-5-1 Chofugaoka, Chofu Tokyo 182-8555, Japan \\ ${ }^{2}$ Department of Electronic and Information Engineering, Chubu University, Kasugai Aichi, Japan \\ ${ }^{3}$ Department of Physics, Kochi University, Kochi Kochi, Japan
}

Received: 23 May 2008 - Revised: 4 June 2008 - Accepted: 5 June 2008 - Published: 17 June 2008

\begin{abstract}
In order to investigate any precursory effect of ionospheric perturbations associated with the large $2007 \mathrm{Ni}-$ igata Chuetsu-oki earthquake (16 July 2007) (magnitude, 6.8), we have made full use of our VLF/LF network observation in Japan by examing the four propagation paths; JJI transmitter (Kyusyu, Ebino)-MSR (Moshiri, Hokkaido), JJY transmitter (Fukushima)-MSR, JJY-KOC (Kochi) and JJICBA (Tateyama, Chiba). For the former two paths of JJIMSR and JJY-MSR, we have observed significant propagation anomalies (both a decrease in nighttime average amplitude and an enhancement in nighttime amplitude fluctuation both satisfying the $2 \sigma$ ( $\sigma$ : standard deviation) criteria) on 89 July, about one week before the earthquake. However, the lack of observation for the path from JJY-KOC, has enabled us to make no conclusion for this path. On the other hand, the path from JJI-CBA does not seem to be perturbed. Although this earthquake is very big and shallow, the amount of VLF anomaly for this earthquake is not so pronounced as expected, probably because the epicenter is not unfortunately located within the sensitive areas of any propagation paths. These observational facts suggest that the lower ionosphere above the epicenter is perturbed definitely prior to the earthquake, with radius of a few hundred kilometers.
\end{abstract}

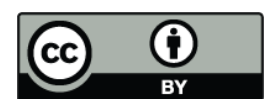

Correspondence to: M. Hayakawa (hayakawa@whistler.ee.uec.ac.jp)

\section{Introduction}

There have been recently accumulated a lot of reports on the electromagnetic precursory effects of earthquakes (EQs) (e.g. Hayakawa, 1999; Molchanov and Hayakawa, 2002, 2007 and 2008). Among these seismo-electromagnetic phenomena, the lower ionosphere has been found to be very sensitive to seismic activity, as based on event and statistical studies by means of subionospheric VLF/LF propagation anomalies (Molchanov and Hayakawa, 2007; Hayakawa and Molchanov, 2008). Event studies include: (1) Kobe-EQ (Hayakawa et al., 1996), (2) 2003 Tokachi-oki EQ (Shvets et al., 2004; Hayakawa et al., 2005), (3) 2004 Niigata Chuetsu EQ (Hayakawa et al., 2006; Yamauchi et al., 2007) and (4) Indonesia Sumatra EQ (Horie et al., 2007a, b). Also, there have been recently statistically confirmed by Rozhnoi et al. (2004) and Maekawa et al. (2006), who have found that the subionospheric VLF/LF propagation anomaly takes place prior to a large (magnitude, at least 5.5) EQ (i.e. (1) amplitude decrease and (2) enhanced nighttime fluctuation).

In this paper we present subionospheric VLF/LF propagation results in order to study, by means of our Japanese VLF/LF network observation, the perturbations in the lower ionosphere prior to a recent large EQ named 2007 Niigata Chuetsu-oki EQ.

\section{Niigata Chuetsu-oki EQ}

The 2007 Niigata Chuetsu-oki EQ happened at 10:13:22 L.T. on 16 July 2007 with the epicenter located at the geographic coordinates $\left(37^{\circ} 33^{\prime} \mathrm{N}, 138^{\circ} 36^{\prime} \mathrm{E}\right)$. Its magnitude was relatively large, $M=6.8$ and its depth was $d=17 \mathrm{~km}$. The place of this EQ is plotted in Fig. 1 as a red large star. The magnitude

Published by Copernicus Publications on behalf of the European Geosciences Union. 


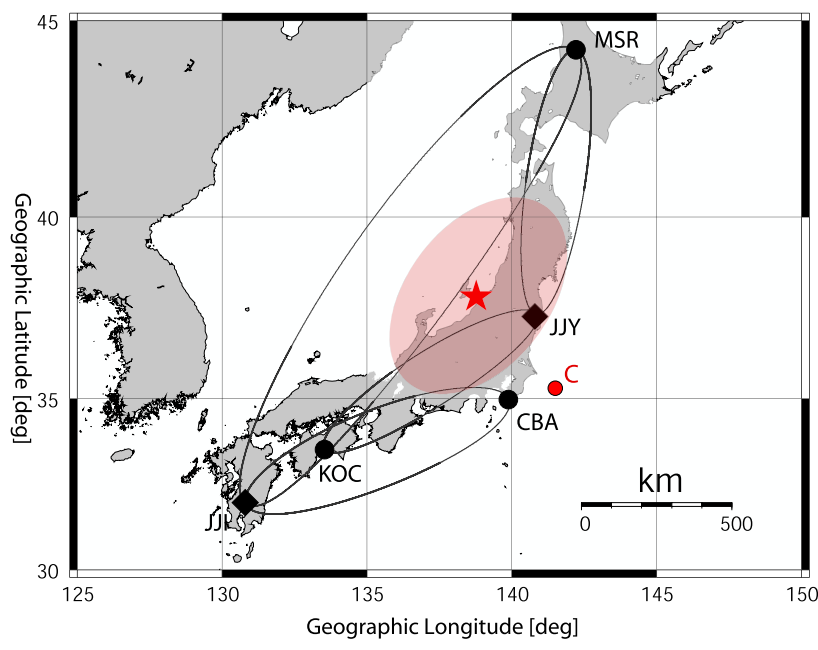

Fig. 1. Four VLF/LF subionospheric propagation paths used for the analysis of seismo-ionospheric perturbations; JJI transmitter (Kyusyu, Ebino)-MSR (Moshiri, Hokkaido), JJI-CBA (Chiba, Tateyama), JJY transmitter (Fukushima)-MSR and JJYKOC (Kochi). The 5th Fresnel zone is plotted for each propagation path. A large red star indicates the epicenter of the 2007 Niigata Chuetsu-oki EQ. Finally, the pink area around the Niigata EQ epicenter, is the possible region of ionospheric perturbation.

of this EQ is extremely large, which is worth studying the related perturbations. However, you will find that there have been seismically active all over Japan during the period we analyzed, so that we need careful examination of the data. The EQ catalogue is taken from USGS.

\section{Subionospheric VLF/LF network observation and data analysis}

Within the framework of the former NASDA's Earthquake Remote Sensing Frontier Project, we established a Japanese VLF/LF network and it has been in operation since 1996 (Hayakawa et al., 2004). The VLF/LF network consists of seven observing stations in Japan: (1) Moshiri (in Hokkaido) (abbreviated as MSR), (2) Chofu, (3) Tateyama (Chiba) (CBA), (4) Shimizu, (5) Kasugai (Nagoya), (6) Maizuru (Kyoto), and (7) Kochi (KOC). Our VLF/LF receiver consists of the VLF and GPS antennas, the VLF pre-amplifier, the Service Unit, and the DSP card for the computer. The receiver can log up to six transmitters at a time, logging phase and amplitude with time resolutions ranging from $50 \mathrm{~ms}$ to $60 \mathrm{~s}$. We receive five VLF/LF transmitter signals (NWC (Australia, $f=19.8 \mathrm{kHz}$ ), NPM (Hawaii, $21.4 \mathrm{kHz}$ ), NLK (USA, $24.8 \mathrm{kHz}$ ), JJY (Fukushima, $40 \mathrm{kHz}$ ) and JJI (Kyushu, Ebino, $22.2 \mathrm{kHz}$ ) ) at each observing station. For the seismo-mode of operation, we use the time resolution of $20 \mathrm{~s}$ and we average the data over $120 \mathrm{~s}$ ( $2 \mathrm{~min}$ ) because the seismo-ionospheric effect is a slow process. The data observed at each station are transmitted to the master station at the University of ElectroCommunications every day. Fig. 1 illustrates the propagation paths used in this paper; JJI-MSR, JJI-CBA, JJY-KOC and JJY-MSR. We have also plotted the 5th Fresnel zone for each path, which means that when any ionospheric perturbed region overlaps with this sensitive zone, we detect a certain anomaly in subionospheric VLF/LF propagation.

The analysis of subionospheric VLF/LF data is described here, though the details of our analysis have already been described in Rozhnoi et al. (2004) and Maekawa et al. (2006). We look at the diurnal variation of amplitude for each path, $A(t)$ ( $t$ : a particular time) on a particular day, and we pay attention to the local nighttime (L.T. $=20 \mathrm{~h} \sim 04 \mathrm{~h}$; UT $=11 \mathrm{~h}$ $\sim 19 \mathrm{~h}$ ). We make a running median (not mean) $\langle A(t)>$ at the time $t$ during \pm 1 month, and we use the following residue,

$\Delta A(t)=A(t)-\langle A(t)\rangle$

in order to deduce the short-term variation by reducing the seasonal effect. Then, we plot, in Fig. 2, (1) average amplitude (trend) and (2) amplitude fluctuation both during local nighttime. Fig. 2 illustrates the temporal evolution of observational results during \pm 2 months around the EQ day (indicated by a vertical red line with EQ) for the relevant propagation paths; from the top, (i) JJI-MSR, (ii) JJY-MSR, (iii) JJY-KOC and (iv) JJI-CBA. For each path there are two plots; upper panel on the nighttime fluctuation and the lower panel, nighttime amplitude trend. Nighttime fluctuation is plotted in arbitrary unit, and the dot-chain line indicates the $2 \sigma$ ( $\sigma$ : standard deviation over \pm 15 days). The corresponding $2 \sigma$ line is given in a blue full line in the lower panel. As is shown by Maekawa et al. (2006), the two physical properties, (1) amplitude decrease at night and (2) enhancement in nighttime fluctuation, are essential peculiarities for seismoionospheric perturbations. In the plots for the path from JJI to MSR, we can identify a definite precursory effect satisfying the criterion of $2 \sigma$ for both the amplitude trend and nighttime fluctuation on 9 July, one week before the EQ. Another anomaly is also seen on 1 July, two weeks before the EQ. Similarly, as is clearly seen from the propagation path from JJI to MSR, there is one sharp peak with amplitude decrease exceeding the $2 \sigma$ criterion and simultaneously with nighttime fluctuation enhancement exceeding $2 \sigma$ line on the day of 8 July, about one week before the EQ. The anomalies for these two paths are extremely conspicuous. Next, we study the results for the propagation path from JJY to KOC. Unfortunately, the observation was not so successful (due to some problems in the observation) during the period from 1 to 20 July including the EQ date, so that we cannot say anything about the information on seismo-ionospheric perturbation for this particular path. Finally, we look at the result for the last propagation path from JJI-CBA in the bottom figure of Fig. 2. There seems to exist no anomalous variations during the period of 1 July to EQ date. 
When discussing the precursory signature of the Niigata Chuetsu-oki EQ, we have to explain other peaks existing in Fig. 2 with satisfying the $2 \sigma$ criteria for both amplitude decrease and enhancement in nighttime amplitude fluctuation. These days are indicated by providing the date of VLF/LF anomaly. We describe those anomalies on each path. First, for the path of JJI-MSR, we find the anomalies on 25 May, 20 August, and 5 September. The 1st anomaly on 25 May is likely to be associated with an EQ in Hokkaido (magnitude 4.5), and the anomaly on May 25 on the path of JJY-MSR is also likely to be the effect of the same EQ. The anomaly on 20 August is probably related to an EQ in Aomori on $25 \mathrm{Au}-$ gust. The anomaly on 5 September or so, will be discussed later. We move on to the next path of JJY-MSR. An EQ in Hakodate on 22 August (magnitude=5.6) is found to exist just after the anomaly on 15 August. Then, the path of JJYKOC is discussed. There is only one anomaly on 15 September, which is likely to be a precursor to the EQ in Ibaraki on 20 September (magnitude=5.1). The last path of JJI-CBA is treated. Before the Niigata-Chuetsu EQ there have been observed three successive VLF anomalies on 25 May, 13 June and 29 June, which are all probably likely to be the effect of clustered EQs in Chiba area (as indicated by $\mathrm{C}$ in Fig. 1) occurred on 4 June (magnitude 4.8), 20 June (magnitude 5.2) and 3 July (magnitude 4.7), respectively. Finally, a huge anomaly in early September nearly on all paths, is highly likely to be associated with a huge meteorological typhoon (\#9 typhoon) which passed the main land of Japan (Honshu) during a few days around the relevant date (5 September). Because Sorokin et al. (2006) have found an evidence of typhoons on the ionospheric perturbations as seen on the satellites.

Here we investigate the geomagnetic activity $(\Sigma \mathrm{Kp}$ and Dst) during the relevant period. Though not shown as a graph, we have found that the value of $\Sigma \mathrm{Kp}$ remained in a range of 5-25 during the whole period (with one exception of a little over 30 on 25 May). So that, we think that the geomagnetic activity has been relatively quiet during the period of our analysis, and this indicates that the effect of geomagnetic disturbances seems to be unlikely.

\section{Discussion}

First of all, we can conclude that some precursory signature of the 2007 Niigata Chuetsu-oki EQ was definitely observed on the two propagation paths form JJI-MSR and JJY-MSR about one week before the EQ. Though the period we analyzed was rather seismically active, so that there were so many EQs all over Japan. The lead time obtained seems to be consistent with our early results by Rozhnoi et al. (2004) and Maekawa et al. (2006).

The VLF/LF propagation anomaly measured in both nighttime fluctuation and trend, is found to be exceeding the corresponding $2 \sigma$ lines, but it seems to be not so conspicuous

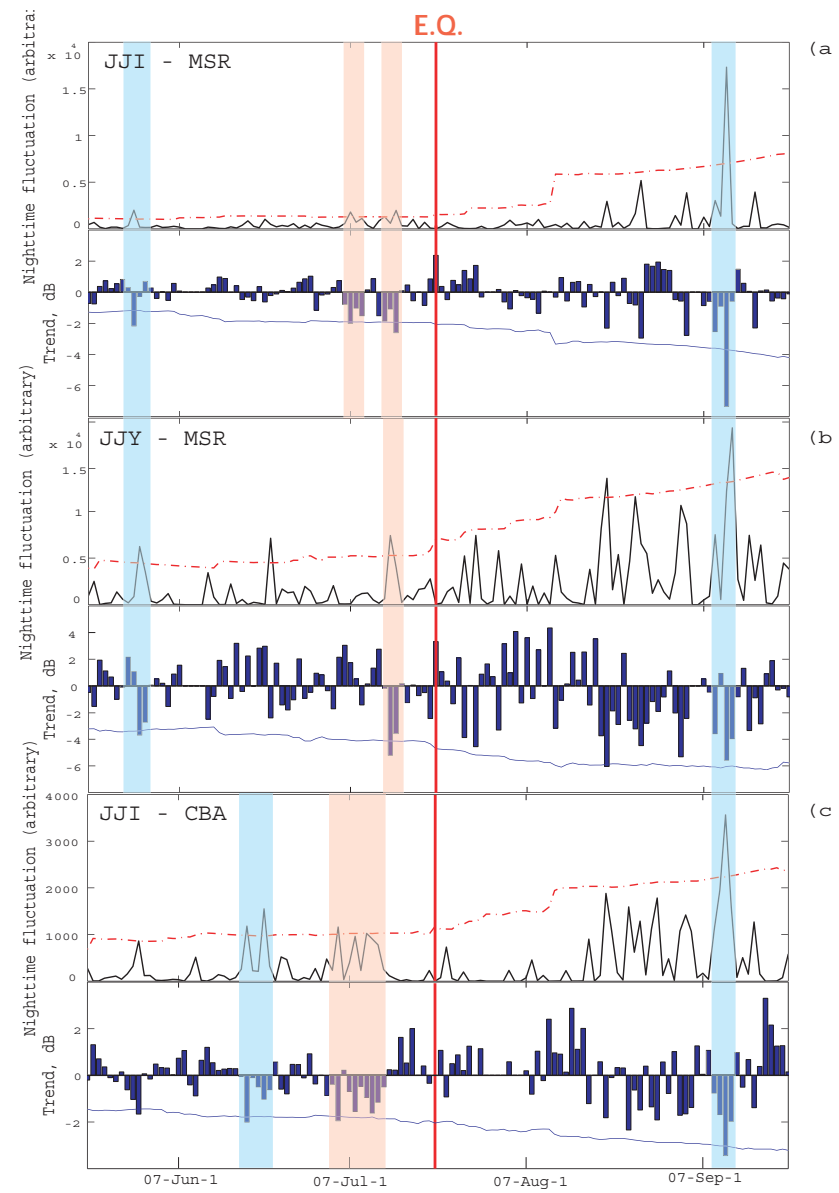

Fig. 2. Temporal evolution of subionospheric VLF/LF propagation characteristics during \pm 2 months around the EQ day (indicated by a red vertical line with EQ). Four propagation paths are indicated; JJI-MSR, JJY-MSR, JJY-KOC and JJI-CBA (from top to bottom). In each plot, there are two plots; upper, nighttime amplitude fluctuation (in arbitrary unit) (with $2 \sigma$ ( $\sigma$ : standard deviation over \pm 15 days) criterion in a dot-broken line), and lower, amplitude trend (+increase, - decrease in amplitude) (with $2 \sigma$ criterion in a blue line). The time region with vertical pink color indicates a possible precursor to the Niigata Chuetsu-oki EQ. C indicates the epicenters of a few clustered EQs in the Chiba area.

as our expectation despite to the huge magnitude of this EQ. This means that the main reason for this might be that the EQ epicenter is located outside the Fresnel zone (wave sensitive area) of any propagation paths. That the EQ epicenter should be located within the Fresnel zone, is of essential significance in obtaining significant propagation anomaly exceeding well above the $2 \sigma$ criterion.

As based on the precursory anomalies definitely observed on the propagation paths from JJI to MSR and from JJY to MSR (and not so definite effect on the path from JJI to CBA), we can infer the region of seismo-ionospheric perturbation, which is plotted in Fig. 1 as a pink area around the EQ epicenter. The red large star indicates the EQ epicenter, 
and we have drawn a nearly circular region. The radius of this seismo- ionospheric perturbation is of the order of a few hundred kilometers. This scale seems to be smaller than that expected by any formula by Dobrovol'sky et al. (1979) or Ruzhin and Depueva (1996), either of which gives us an approximate value of $R \cong 900 \mathrm{~km}$ for the given magnitude. The experimental value is of the order of one third of these expectations. However, this observational scale is found to be very similar to that for 2004 Niigata Chuetsu EQ (Yamauchi et al., 2007).

The purpose of this paper is to present the observational evidence (i.e. presence or absence, lead time, spatial scale or so) on the ionospheirc perturbations associated with the recent relatively large 2007 Niigata Chuetsu-oki EQ (magnitude 6.8), so that we do not go into the detailed discussion on the lithosphere-ionosphere coupling mechanism. Please refer to Hayakawa and Molchanov (2007) or Molchanov and Hayakawa (2008), in which there have been proposed a few possible coupling mechanisms; (1) chemical channel, in which the atmospheric electric field plays an important role in the coupling and (2) acoustic channel, in which the atmospheric oscillations (e.g. atmospheric gravity waves) play an essential role in the coupling mechanism (Molchanov et al., 2001).

\section{Conclusions}

The Japanese VLF/LF network observation has enabled us to identify definitely the presence of ionospheric anomaly related to the huge 2007 Niigata EQ and also to indicate its spatial scale.

Acknowledgements. This work was partly supported by NiCT ( R and $\mathrm{D}$ promotion scheme funding international joint research), to which we are grateful.

Edited by: M. Contadakis

Reviewed by: P. F. Biagi and A. Rozhnoi

\section{References}

Dobrovol'sky, I. P., Zubkov, S. I., and, Miachkin, V. I.: Estimation of the size of earthquake preparation zones, 117, 1025-1044, 1979.

Hayakawa, M., Molchanov, O. A., Ondoh, T., and Kawai, E.: The precursory signature effect of the Kobe earthquake on VLF subionospheric signals, J. Comm. Res. Lab., Tokyo, 43, 169$180,1996$.

Hayakawa, M.: Atmospheric and Ionospheric Electromagnetic Phenomena Associated with Earthquakes, Terra Sci. Pub. Co., 996p, Tokyo, 1999.

Hayakawa, M. and Molchanov, O. A. Editors, Seismo Electromagnetics: Lithosphere - Atmosphere - Ionosphere Coupling, TERRAPUB, 477p, Tokyo, 2002.
Hayakawa, M., Molchanov, O. A. and NASDA/UEC team, Summary report of NASDA's earthquake remote sensing frontier project, Phys. Chem. Earth, 29, 617-625, 2004.

Hayakawa, M., Shvets, A. V., and Maekawa, S.: Subionospheric LF monitoring of ionospheric perturbations prior to the Tokachi-oki earthquake and a possible mechanism to lithosphere - ionosphere coupling, Adv. Polar Upper Atmos. Res., 19, 42-54, 2005.

Hayakawa, M., Ohta, K., Maekawa, S., Yamauchi, T., Ida, Y., Gotoh, T., Yonaiguchi, N., Sasaki, H., and Nakamura, T.: Electromagnetic precursors to the 2004 Mid Niigata Prefecture earthquake, Phys. Chem. Earth,31, 356-364, 2006.

Hayakawa, M. and O. A., Molchanov, Seismo-electromagnetics as a new field of radiophysics: Electromagnetic phenomena associated with earthquakes, Radio Sci. Bull., 320, 8-17, 2007.

Horie, T., Maekawa, S., Yamauchi, T., and Hayakawa, M.: A possible effect of ionospheric perturbations associated with the Sumatra earthquake, as revealed from subionospheric very-lowfrequency (VLF) propagation (NWC-Japan), Int'1 J. Remote Sensing, 28, 13, 3133-3139, 2007a.

Horie, T., Yamauchi, T., Yoshida, M., and Hayakawa, M.: The wave-like structures of ionospheric perturbation associated with Sumatra earthquake of 26 December 2004, as revealed from VLF observation in Japan of NWC signals, J. Atmos. Solar-terr. Phys., 69, 1021-1028, 2007b.

Maekawa, S., Horie, T., Yamauchi, T., Sawaya, T., Ishikawa, M., Hayakawa, M., and Sasaki,H.: A statistical study on the effect of earthquakes on the ionosphere, based on the subionospheric LF propagation data in Japan, Ann. Geophysicae, 24, 2219-2225, 2006.

Molchanov, O. A., Hayakawa, M., and Miyaki, K.: VLF/LF sounding of the lower ionosphere to study the role of atmospheric oscillations in the lithosphere-ionosphere coupling, Adv. Polar Upper Atmos. Res., 15,146-158, 2001.

Molchanov, O. A., and Hayakawa, M.: Seismo-electromagnetics and Related Phenomena: History and Latest Results, TERRAPUB, Tokyo, 189p., 2008.

Rozhnoi, A., Solovieva, M. S.,Molchanov, O. A., and Hayakawa, M.: Middle latitude LF $(40 \mathrm{kHz})$ phase variations associated with earthquakes for quiet and disturbed geomagnetic conditions, Phys. Chem. Earth, 29, 589-598, 2004.

Ruzhin, Y. Y. and Depueva, K. A.: Seismoprecursors in space as plasma and wave anomalies, J. Atmos. Electr., 16, 271-288, 1996.

Shvets, A. V., Hayakawa, M., and Maekawa, S.: Results of subionospheric radio LF monitoring prior to the Tokachi $(\mathrm{m}=8$, Hokkaido, 25 September 2003) earthquake, Natural Hazards Earth System Sci., 4, 647-653, 2004.

Sorokin, V. M., Yaschenko, A. K., Chmyrev, V. M., and Hayakawa, M.: DC electric field formation in the mid-latitude ionosphere over typhoon and earthquake regions, Phys. Chem. Earth, 31, 454-461, 2006.

Yamauchi, T., Maekawa, S., Horie, T., Hayakawa, M., and Soloviev, O.: Subionospheric VLF/LF monitoring of ionospheric perturbations for the 2004 Mid-Niigata earthquake and their structure and dynamics, J. Atmos. Solar-terr. Phys., 69, 793-802, 2007. 